\title{
Isoperimetric Quotients for a Decomposed Convex Body
}

\author{
G. Blind ${ }^{1}$ and R. Blind ${ }^{2}$ \\ ${ }^{1}$ Mathematisches Institut B, Universität Stuttgart, \\ Pfaffenwaldring 57, D-70550 Stuttgart, Germany \\ 2 Waldburgstrasse 88, D-70563 Stuttgart, Germany
}

\begin{abstract}
In the Euclidean plane, decompose a convex body $T$ into $n \geq 2$ convex bodies $T_{1}, \ldots, T_{n}$ with areas also denoted by $T_{1}, \ldots, T_{n}$, and with perimeters $L_{1}, \ldots, L_{n}$. For $T$ a polygon with at most six sides, G. Fejes Tóth and also L. Fejes Tóth showed that the isoperimetric quotient $\left(L_{1}+\cdots+L_{n}\right) /\left(\sqrt{T}_{1}+\cdots+\sqrt{T}_{n}\right)$ is greater than the corresponding isoperimetric quotient of a regular hexagon if $T_{i} / T_{j}$ for any $i, j$ is bounded from below by some appropriate constant. We generalize this result to any convex body $T$, and we show the analogous result for the isoperimetric quotient $\left(L_{1}^{2}+\cdots+L_{n}^{2}\right) /\left(T_{1}+\cdots+T_{n}\right)$.
\end{abstract}

\section{Introduction}

In the Euclidean plane, decompose a convex body $T$ into $n \geq 2$ convex bodies $T_{1}, \ldots, T_{n}$ with areas denoted also by $T_{1}, \ldots, T_{n}$, and with perimeters $L_{1}, \ldots, L_{n}$. We are interested in the isoperimetric quotients of these quantities. Recall that the perimeter of a regular $p$-gon of area 1 is $h(p)=2 \sqrt{p \tan (\pi / p)}$, and define $g(p):=4 p \tan (\pi / p)=h^{2}(p)$. In this paper we show

\section{Theorem 1.}

$$
\frac{L_{1}+\cdots+L_{n}}{\sqrt{T_{1}}+\cdots+\sqrt{T_{n}}}>2 \sqrt[4]{12}=h(6)=3.7224 \cdots
$$

if

$$
\frac{\min \sqrt{T_{i}}}{\max \sqrt{T_{i}}} \geq \frac{h(6)-h(7)}{h(5)-h(6)}=: h_{0}=0.562477 \ldots
$$




\section{Theorem 2.}

$$
\frac{L_{1}^{2}+\cdots+L_{n}^{2}}{T_{1}+\cdots+T_{n}}>4 \sqrt{12}=g(6)=13.8564 \cdots
$$

if

$$
\frac{\min T_{i}}{\max T_{i}} \geq \frac{g(6)-g(7)}{g(5)-g(6)}=: g_{0}=0.552035 \cdots
$$

Thus, both isoperimetric quotients are greater than the corresponding isoperimetric quotient of a regular hexagon if the areas $T_{1}, \ldots, T_{n}$ are not too different.

Note that the bounds given in (1.1) and (1.3) are best possible, since each $T$ for sufficiently large $n$ admits a decomposition into $n$ convex bodies such that the isoperimetric quotients in (1.1) and (1.3) are arbitrarily close to the given bounds.

However, for small $n$ better bounds exist. For each given $n$, let $Q(n)$ and $\bar{Q}(n)$ be the minimum of the isoperimetric quotients in (1.1) and (1.3), respectively, for all convex $T$ and all decompositions into $n$ convex bodies. Then our proofs of Theorems 1 and 2 also yield

\section{Theorem 3.}

(i) $Q(2)>3.7614$ and $Q(3)>3.7616$.

(ii) $\bar{Q}(2)>14.1982$ and $\bar{Q}(3)>14.1744$.

Until now Theorems 1 and 2 as well as related results were only known to be true for convex polygons $T$ with at most six sides. G. Fejes Tóth [2] and also L. Fejes Tóth [4] proved (1.1) under the same condition as (1.2) in the particular case that $T$ is a convex polygon with at most six sides. A condition of type (1.2) is necessary, since an example is given in [5] where $\min \sqrt{T_{i}} / \max \sqrt{T_{i}}<h_{0}$, and (1.1) is not valid. However, the question whether $h_{0}$ can be replaced by a smaller constant is open.

If $\min \sqrt{T_{i}} / \max \sqrt{T_{i}} \geq q$ for some $q$ with $0<q<h_{0}$, a lower bound $I(q)<2 \sqrt[4]{12}$ for the isoperimetric quotient in (1.1) is given in [7] if $T$ is a convex polygon with at most six sides.

As to (1.3), a condition of type (1.4) is necessary, as the example in [5] also shows. The question whether $g_{0}$ can be replaced by a smaller constant is open.

L. Fejes Tóth [4] proved (1.3) in the particular case that $T$ is a convex polygon with at most six sides, but he used the different condition $\min L_{i}^{2} / \max L_{i}^{2} \geq 0.5948 \cdots$. If $\min L_{i}^{2} / \max L_{i}^{2} \geq q$ for some $q$ with $0<q<0.5948 \cdots$, a lower bound $I^{\prime}(q)<4 \sqrt{12}$ for the isoperimetric quotient in (1.3) is given in [6] if $T$ is a convex polygon with at most six sides.

The improvement obtained by dealing with general convex bodies $T$ instead of only convex polygons with at most six sides is illustrated by the following.

For each given $n$, let $Q_{6}(n)$ and $\bar{Q}_{6}(n)$ be the minimum of the isoperimetric quotients in (1.1) and (1.3), respectively, if for $T$ only convex polygons with at most six sides are admitted. While Theorem 1 together with the above reasoning shows that $Q(n)$ and $Q_{6}(n)$ are asymptotically the same, they differ considerably for small $n$. For example, Fig. 1 shows two decompositions constructed by means of circles, whose 

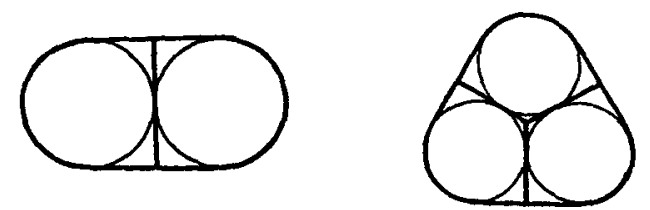

Fig. 1. Two decompositions of convex domains constructed by means of circles.

isoperimetric quotients are easily calculated and yield upper bounds for $Q(2)$ and $Q(3)$, namely, $Q(2) \leq 3.7793 \cdots$ and $Q(3) \leq 3.7725 \cdots$. On the other hand, it is easily seen (though not trivial) that the rough lower bounds $h(5)=3.8119 \cdots \leq Q_{6}(2)$ and $h(5)=3.8119 \ldots \leq Q_{6}(3)$ hold. Analogously we obtain $\bar{Q}(2) \leq 14.2831 \ldots$ and $\bar{Q}(3) \leq 14.2579 \cdots$, and on the other hand $g(5)=14.5308 \cdots \leq \bar{Q}_{6}(2)$ and $g(5)=14.5308 \cdots \leq \bar{Q}_{6}(3)$. Thus, for small $n$, general convex bodies allow much smaller isoperimetric quotients than hexagons, and so the bounds in Theorems 1 and 2 are much sharper for general convex bodies than they are for hexagons. obtain

Collecting the upper bounds given above and the lower bounds of Theorem 3 , we

$$
\begin{aligned}
& 3.7793 \cdots \geq Q(2)>3.7614 \text { and } 3.7725 \cdots \geq Q(3)>3.7616 \\
& 14.2831 \cdots \geq \bar{Q}(2)>14.1982 \text { and } 14.2579 \cdots \geq \bar{Q}(3)>14.1744
\end{aligned}
$$

We prove Theorems 1 and 3(i) in Sections 2-7. Using the same techniques, we prove Theorems 2 and 3(ii) in Section 8.

\section{Basic Considerations}

Without loss of generality we may assume that $T$ is smooth, strictly convex, and that the vertices of the decomposition of $T$ are all 3 -valent. The number of such vertices is $2 n-2$ by Euler's theorem.

If a domain $T_{i}(1 \leq i \leq n)$ contains boundary points of $T$, then $T_{i}$ is an exterior domain; otherwise, $T_{i}$ is an interior domain. Let $e$ be the number of exterior domains. Each exterior domain having at least two vertices on the boundary of $T$, the number of vertices on the boundary of $T$ is $e+\mathcal{E}$ for some integer $\mathcal{E} \geq 0$. Let $p_{i}$ be the vertex number of $T_{i}$. Then $p_{1}+\cdots+p_{n}+e+\mathcal{E}=3(2 n-2)$. Thus

$$
p_{1}+\cdots+p_{n}=6 n-6-e-\mathcal{E} \quad \text { for some integer } \quad \mathcal{E} \geq 0 \text {. }
$$

Denote the exterior angles of the vertices of $T_{i}$ by $\beta_{1}^{i}, \ldots, \beta_{p_{i}}^{i}$, see Fig. 2. Put $y_{i}:=$ $\sum_{k=1}^{p_{i}} \frac{1}{2} \beta_{k}^{i}$. Then $y_{i}<\pi$ for exterior domains $T_{i}$, and $y_{i}=\pi$ for interior domains $T_{i}$. Clearly,

$$
y_{1}+\cdots+y_{n}=n \pi-\pi \text {. }
$$

Note that $p_{i} \geq 2$, and that $p_{i}=2$ is only possible for an exterior domain, that is, for $y_{i}<\pi$. Thus $y_{i} / p_{i}<\pi / 2$. 


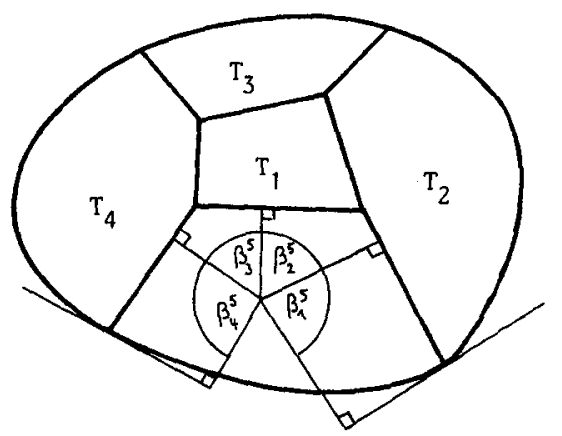

Fig. 2. The exterior angles of the vertices of $T_{i}$.

Using Lhuilier's inequality, it is shown in [1] that

$$
\frac{L_{i}}{\sqrt{T_{i}}} \geq \frac{2}{\sqrt{\pi}}\left(\pi-y_{i}+\sqrt{p_{i} y_{i} \tan \frac{y_{i}}{p_{i}}}\right) \text {. }
$$

With the function

$$
H(p, y):=\frac{2}{\sqrt{\pi}}\left(\pi-y+\sqrt{p y \tan \frac{y}{p}}\right)
$$

defined on the convex domain $D$ given by $p>0,0 \leq y \leq \pi$, and $y / p<\pi / 2$, we may rewrite (2.3) as

$$
L_{i} \geq \sqrt{T_{i}} H\left(p_{i}, y_{i}\right)
$$

Thus, in order to prove Theorem 1 , it is enough to show, with $c:=2 \sqrt[4]{12}$,

$$
\sqrt{T_{1}}\left[H\left(p_{1}, y_{1}\right)-c\right]+\cdots+\sqrt{T_{n}}\left[H\left(p_{n}, y_{n}\right)-c\right]>0,
$$

where $\left(p_{i}, y_{i}\right)$ are points of $D$ satisfying (2.1) and (2.2), and because of (1.2) we may add the condition

$$
h_{0} \leq \sqrt{T_{i}} \leq 1 \quad \text { for } \quad 1 \leq i \leq n
$$

\section{The Function $H(p, y)$}

We deduce some properties of the function $H(p, y)$ with domain $D$. An easy calculation shows that, for $(p, \pi) \in D$ and for any $t$ with $0<t \leq 1$,

$$
H(t p, t \pi)=2 \sqrt{\pi}+t[H(p, \pi)-2 \sqrt{\pi}],
$$

which is a linear function of $t$, and can be extended to $t=0$. Thus, in a $(p, y, z)-$ coordinate system of $\mathbb{R}^{3}$, the function $z=H(p, y)$ describes a cone with apex $(0,0,2 \sqrt{\pi})$ and with straight line segments lying in the planes $y / p=$ constant. This implies, for the partial derivatives,

$$
H_{p}(t p, t \pi)=H_{p}(p, \pi) \quad \text { and } \quad H_{y}(t p, t \pi)=H_{y}(p, \pi) .
$$

Intersecting the cone $z=H(p, y)$ with the plane $y=\pi$, we obtain in this plane the curve $z=H(p, \pi)=h(p)$ (see Section 1). As was shown in [3], this is a convex function for $p>2$. So $H(p, y)$ is a convex function. 
Calculating the partial derivatives of $H(p, y)$, we easily see that

$$
H_{p}(p, y) \leq 0 \text { and } H_{y}(p, y)>0 \text { for all }(p, y) \in D
$$

Note that

$$
H(6, \pi)=h(6)=c .
$$

Moreover, the definition of $h_{0}$ in (1.2) is such that

$$
[H(7, \pi)-c]=-h_{0}[H(5, \pi)-c]
$$

\section{Using the Convexity of $H(p, y)$}

Next, we estimate the left side of $(2.5)$ by a simpler function. Using the convexity of $H(p, y)$, we show

Lemma 4.1. Under the conditions (2.1), (2.2), and (2.6), the left side of (2.5) can be estimated as follows:

(a) For the nonnegative summands in (2.5) with $y_{i}<\pi$, we have

$$
\sum \sqrt{T_{i}}\left[H\left(p_{i}, y_{i}\right)-c\right] \geq u h_{0}\left[H\left(p_{u}, y_{u}\right)-c\right]
$$

where $u \geq 0$ is the number of such summands, and $p_{u}$ and $y_{u}$ are the mean values $p_{u}=(1 / u) \sum p_{i}$ and $y_{u}=(1 / u) \sum y_{i}$.

(b) For the negative summands in (2.5) with $y_{i}<\pi$, we have

$$
\sum \sqrt{T_{i}}\left[H\left(p_{i}, y_{i}\right)-c\right] \geq v\left[H\left(p_{v}, y_{v}\right)-c\right],
$$

where $v \geq 0$ is the number of such summands, and $p_{v}$ and $y_{v}$ are the mean values $p_{v}=(1 / v) \sum p_{i}$ and $y_{v}=(1 / v) \sum y_{i}$.

(c) For the summands in (2.5) with $y_{i}=\pi$, we have

$$
\sum \sqrt{T_{i}}\left[H\left(p_{i}, y_{i}\right)-c\right] \geq\left(u p_{u}+v p_{v}-5(u+v)+6\right) h_{0}[H(5, \pi)-c] .
$$

Moreover,

$$
u y_{u}+v y_{v}=(u+v-1) \pi,
$$

and $u+v$ is the number of exterior domains of our decomposition.

Proof. Because of (2.6), for each summand of (2.5) we have

$$
\sqrt{T_{i}}\left[H\left(p_{i}, y_{i}\right)-c\right] \geq \begin{cases}h_{0}\left[H\left(p_{i}, y_{i}\right)-c\right] & \text { if }\left[H\left(p_{i}, y_{i}\right)-c\right] \geq 0 \\ {\left[H\left(p_{i}, y_{i}\right)-c\right]} & \text { if }\left[H\left(p_{i}, y_{i}\right)-c\right]<0\end{cases}
$$

Then (a) and (b) follow immediately from the convexity of $H(p, y)$, and (4.2) follows from (2.2) and the definition of $y_{u}$ and $y_{v}$. Moreover, recall that the summands in (a) 
and (b) correspond to the exterior domains of our decomposition. Thus, their number $e$ is $e=u+v$, and (2.1) becomes

$$
u p_{u}+v p_{v}+\sum p_{i}=6 n-6-(u+v)-\mathcal{E} \quad \text { for some } \quad \mathcal{E} \geq 0
$$

where $\sum p_{i}$ refers to the points $\left(p_{i}, \pi\right)$ occurring in (c).

In order to show $(c)$, note that summands with $\left(p_{i}, \pi\right)=(6, \pi)$ are zero. So omit the points $(6, \pi)$ and accordingly change $n$; thereby condition (4.3) continues to hold.

Note that (3.3), (3.4), and the convexity of $H(p, y)$ imply $[H(4, \pi)-c]=[H(4, \pi)-$ $H(5, \pi)]+[H(5, \pi)-c] \geq 2[H(5, \pi)-c]$, and, in general,

$$
\left[H\left(p_{i}, \pi\right)-c\right] \geq\left(6-p_{i}\right)[H(5, \pi)-c] \quad \text { for } \quad p_{i} \leq 4 .
$$

Analogously, we have

$$
\left[H\left(p_{i}, \pi\right)-c\right] \geq\left(p_{i}-6\right)[H(7, \pi)-c] \quad \text { for } \quad p_{i} \geq 8 .
$$

We substitute each point $\left(p_{i}, \pi\right)$ with $p_{i} \leq 4$ by $\left(6-p_{i}\right)$ points $(5, \pi)$, and we substitute each point $\left(p_{i}, \pi\right)$ with $p_{i} \geq 8$ by $\left(p_{i}-6\right)$ points $(7, \pi)$. Then $(4.3)$ continues to hold if $n$ is accordingly changed.

Thus, we now deal with a certain number $a \geq 0$ of points $(5, \pi)$ and a certain number $b \geq 0$ of points $(7, \pi)$, and (4.3) now reads $u p_{u}+v p_{v}+a 5+b 7=6(a+b+u+v)-$ $6-(u+v)-\mathcal{E}$, or

$$
a-b=u p_{u}+v p_{v}-5(u+v)+6+\mathcal{E} .
$$

Moreover, by (4.4) and (4.5), for the summands in (2.5) with $y_{i}=\pi$ we have $\sum \sqrt{T_{i}}\left[H\left(p_{i}, \pi\right)-c\right] \geq a h_{0}[H(5, \pi)-c]+b[H(7, \pi)-c]=(a-b) h_{0}[H(5, \pi)-c]$, by (3.5). Substituting $(a-b)$ by (4.6) and omitting the term $\mathcal{E} h_{0}[H(5, \pi)-c] \geq 0$ proves (c).

Remark 4.2. If, in particular, a triangular interior domain occurs, that is, a point $\left(p_{i}, \pi\right)=(3, \pi)$ occurs in (c), then the estimation (4.4) neglects the quantity 0.568 . Thus in this case we may add $h_{0} 0.568=0.319$ to the right side of (4.1).

\section{Proof of Theorem 1 in the Case of at Least Four Exterior Domains}

Assume that, in the decomposition of $T$, at least four exterior domains occur. By Lemma 4.1, in order to show (2.5) in this case, it is enough to show

$$
\begin{aligned}
F\left(u, v ; p_{u}, p_{v}, y_{u}, y_{v}\right):= & u h_{0}\left[H\left(p_{u}, y_{u}\right)-c\right]+v\left[H\left(p_{v}, y_{v}\right)-c\right] \\
& +\left(u p_{u}+v p_{v}-5(u+v)+6\right) h_{0}[H(5, \pi)-c] \\
> & 0,
\end{aligned}
$$

for all integers $u \geq 0$ and $v \geq 0$ with $u+v \geq 4$, and for all points $\left(p_{u}, y_{u}\right)$ and $\left(p_{v}, y_{v}\right)$ of the domain $D$ with

$$
u y_{u}+v y_{v}=(u+v-1) \pi .
$$


First, consider the case when $u, v \neq 0$, that is, $u, v \geq 1$. Note that $\left(p_{v}, y_{v}\right) \in D$ yields $y_{v} \leq \pi$, and so (5.2) yields $y_{u} \geq((u-1) / u) \pi$. We introduce a new variable $t$ by $y_{u}:=t \pi$, with $(u-1) / u \leq t \leq 1$. Then $y_{v}=((u+v-1-u t) / v) \pi$ by (5.2). Thus, instead of the two variables $y_{u}$ and $y_{v}$ linked by (5.2) we now deal with $t$. Then (5.1) under the condition (5.2) is equivalent to

$$
\begin{aligned}
F\left(u, v ; p_{u}, p_{v}, t\right):= & u h_{0}\left[H\left(p_{u}, t \pi\right)-c\right]+v\left[H\left(p_{v}, \frac{u+v-1-u t}{v} \pi\right)-c\right] \\
& +\left(u p_{u}+v p_{v}-5(u+v)+6\right) h_{0}[H(5, \pi)-c]>0 .
\end{aligned}
$$

The derivative of $F$ with respect to $p_{u}$ is

$$
\frac{\partial F}{\partial p_{u}}=u h_{0} H_{p}\left(p_{u}, t \pi\right)+u h_{0}[H(5, \pi)-c] .
$$

As is easily checked, $H_{p}(5.4635 \cdots, \pi)+[H(5, \pi)-c]=0$, and so (3.2) yields $H_{p}(t 5.4635 \cdots, t \pi)+[H(5, \pi)-c]=0$. Therefore, $\partial F / \partial p_{u}=0$ for $p_{u}=t 5.4635 \cdots$. Moreover, this value for $p_{u}$ in fact yields the minimum of $F$ for given $u, v, p_{v}$, and $t$, since $H\left(p_{u}, \cdot\right)$ is a convex function of $p_{u}$. Substituting this value for $p_{u}$ in (5.3), we must show that

$$
\begin{aligned}
F\left(u, v ; p_{v}, t\right):= & u h_{0}[H(t 5.4635 \cdots, t \pi)-c]+v\left[H\left(p_{v}, \frac{u+v-1-u t}{v} \pi\right)-c\right] \\
& +\left(u t 5.4635 \cdots+v p_{v}-5(u+v)+6\right) h_{0}[H(5, \pi)-c] \\
> & 0 .
\end{aligned}
$$

The derivative of this function $F$ with respect to $p_{v}$ is

$$
\frac{\partial F}{\partial p_{v}}=v H_{p}\left(p_{v}, \frac{u+v-1-u t}{v} \pi\right)+v h_{0}[H(5, \pi)-c]
$$

As is easily checked, $H_{p}(6.4708 \cdots, \pi)+h_{0}[H(5, \pi)-c]=0$. By means of (3.2), it follows as above that $F$ attains its minimum for $p_{v}=((u+v-1-u t) / v) 6.4708 \cdots$. Substituting this in (5.4) and rewriting the result by means of (3.1), we must show that

$$
\begin{aligned}
F(u, v ; t):= & u h_{0}[2 \sqrt{\pi}+t[H(5.4635 \cdots, \pi)-2 \sqrt{\pi}]-c] \\
& +v\left[2 \sqrt{\pi}+\frac{u+v-1-u t}{v}[H(6.4708 \cdots, \pi)-2 \sqrt{\pi}]-c\right] \\
& +(u t 5.4635 \cdots+(u+v-1-u t) 6.4708 \cdots-5(u+v)+6) \\
& \times h_{0}[H(5, \pi)-c]>0 .
\end{aligned}
$$

This function $F$ is linear in $t$ and decreasing in $t$. Thus $F$ attains its minimum for $t=1$. Substituting this in (5.5), we must show that

$$
u 0.0463+v 0.0471-0.1743>0,
$$

which is, in fact, true for $u+v \geq 4$. 
In the case where $u=0$, it follows from (5.2) that $y_{v}=((v-1) / v) \pi$. Thus we must show that

$$
F\left(v, p_{v}\right):=v\left[H\left(p_{v}, \frac{v-1}{v} \pi\right)-c\right]+\left(v p_{v}-5 v+6\right) h_{0}[H(5, \pi)-c]>0 \text {. }
$$

The derivative of $F$ with respect to $p_{v}$ is $\partial F / \partial p_{v}=v H_{p}\left(p_{v},((v-1) / v) \pi\right)+$ $v h_{0}[H(5, \pi)-c]$. Thus, as above, $F$ attains its minimum for $p_{v}=((v-1) / v) 6.4708 \cdots$. Substituting this in (5.6) and rewriting the result by means of (3.1), we obtain

$$
F\left(v, p_{v}\right) \geq v 0.0471-0.1743
$$

which is, in fact, positive for $v \geq 4$.

In the case where $v=0$, we obtain in the same way

$$
F\left(u, p_{u}\right) \geq u 0.0463-0.0959
$$

which is, in fact, positive for $u \geq 4$.

\section{Proof of Theorem 1 in the Case of Three Exterior and at Least One Interior Domains}

We also use (2.5) and Lemma 4.1, but we involve some specific information about $p_{u}$ and $p_{v}$, and some specific information about the interior domains.

Label the domains of the decomposition of $T$ in such a way that $T_{1}, T_{2}, T_{3}$ are the three exterior domains, with vertex numbers $p_{1}, p_{2}, p_{3}$, respectively. We assume that at least one interior domain occurs. Then, using the convexity of the domains, it is easily seen that $p_{1}, p_{2}, p_{3} \geq 4$, and that $p_{1}+p_{2}+p_{3}=12$ or $p_{1}+p_{2}+p_{3} \geq 14$, see Fig. 3 .

Considering the interior domains more closely, we first tighten the estimations in Section 2 leading to (2.5). Since precisely three exterior domains exist, precisely three vertices of the decomposition lie in two exterior and one interior domains (see Fig. 3). The respective angles of the interior domains have a sum of at most $\pi$, so at least one such angle is not greater than $\pi / 3$. Thus an interior domain exists, say $T_{4}$, with an exterior angle, say $\beta_{1}^{4}$, such that $\beta_{1}^{4} \geq 2 \pi / 3$. We use this to tighten (2.3) for $i=4$.
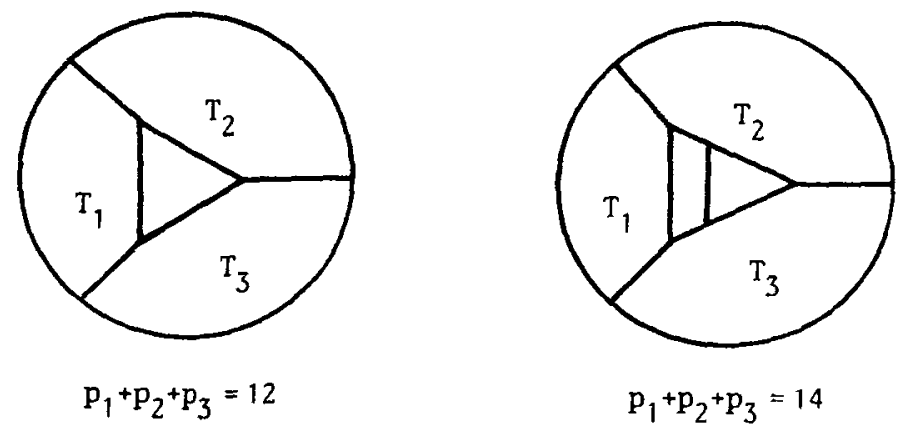

Fig. 3. The topological types of all the decompositions with three exterior and one or two interior domains. 
Lhuilier's inequality (see, e.g., p. 10 of [3]) yields

$$
\frac{L_{4}}{\sqrt{T_{4}}} \geq 2 \sqrt{\sum_{k=1}^{p_{4}} \tan \frac{1}{2} \beta_{k}^{4}}
$$

and so, by Jensen's inequality and $\sum_{k=1}^{p_{4}} \frac{1}{2} \beta_{k}^{4}=\pi$,

$$
\frac{L_{4}}{\sqrt{T_{4}}} \geq 2 \sqrt{\tan \frac{1}{2} \beta_{1}^{4}+\left(p_{4}-1\right) \tan \frac{1}{p_{4}-1}\left(\pi-\frac{1}{2} \beta_{1}^{4}\right)}
$$

Differentiation shows that this is an increasing function of $\beta_{1}^{4}$ for $\beta_{1}^{4} \geq 2 \pi / p_{4}$. Because $p_{4} \geq 3$ and $\beta_{1}^{4} \geq 2 \pi / 3, \beta_{1}^{4}$ satisfies this condition, and thus

$$
\frac{L_{4}}{\sqrt{T_{4}}} \geq 2 \sqrt{\tan \frac{\pi}{3}+\left(p_{4}-1\right) \tan \frac{2 \pi}{3\left(p_{4}-1\right)}} .
$$

The difference between this estimation and that of $(2.3)$ is

$$
\Delta\left(p_{4}\right):=2 \sqrt{\tan \frac{\pi}{3}+\left(p_{4}-1\right) \tan \frac{2 \pi}{3\left(p_{4}-1\right)}}-2 \sqrt{p_{4} \tan \frac{\pi}{p_{4}}} .
$$

Differentiation yields that both terms are decreasing in $p_{4}$, and the first term tends to $3.9122 \cdots$ for $p_{4} \rightarrow \infty$. Using this and evaluating both terms for $p_{4}=3, \ldots, 6$, one obtains

$$
\Delta\left(p_{4}=3\right)=0 \text { and } \Delta\left(p_{4} \geq 4\right) \geq 0.122 \text {. }
$$

Thus, for $p_{4} \geq 4$, we have $L_{4} \geq \sqrt{T_{4}}\left(H\left(p_{4}, \pi\right)+0.122\right) \geq \sqrt{T_{4}} H\left(p_{4}, \pi\right)+0.068$ because $\sqrt{T_{4}} \geq h_{0}$. So, instead of (2.5), it is enough to show

$$
\sum_{i=1}^{3} \sqrt{T_{i}}\left[H\left(p_{i}, y_{i}\right)-c\right]+ \begin{cases}\sum_{i=4}^{n} \sqrt{T_{i}}\left[H\left(p_{i}, \pi\right)-c\right]>0 & \text { if } p_{4}=3 \\ \sum_{i=4}^{n} \sqrt{T_{i}}\left[H\left(p_{i}, \pi\right)-c\right]+0.068>0 & \text { otherwise. }\end{cases}
$$

Of the terms $\sqrt{T_{i}}\left[H\left(p_{i}, y_{i}\right)-c\right]$ for $i=1,2,3$, omit the positive ones, and use Lemma 4.1(b) and (c) and Remark 4.2. Thus it is enough to show that, for $u+v=3$,

$$
\begin{aligned}
& v\left[H\left(p_{v}, y_{v}\right)-c\right]+\left(u p_{u}, v p_{v}-9\right) h_{0}[H(5, \pi)-c] \\
& + \begin{cases}0.319>0 & \text { if } p_{1}+p_{2}+p_{3}=12, \\
0.068>0 & \text { otherwise }\end{cases}
\end{aligned}
$$

where

$$
u y_{u}+v y_{v}=2 \pi
$$

In order to prove (6.5) under condition (6.6), recall that

$$
u p_{u}+v p_{v}=p_{1}+p_{2}+p_{3}=12 \text { or } 14+k \quad \text { for } k=0,1,2, \ldots
$$


Then the sum of the last two terms of (6.5) is

$$
\begin{aligned}
(12-9) 0.050+0.319 & =0.469 & \text { for } & p_{1}+p_{2}+p_{3}=12 \\
(14+k-9) 0.050+0.068 & =0.318+k 0.050 & \text { for } & p_{1}+p_{2}+p_{3}=14+k
\end{aligned}
$$

Thus (6.5) is true for $v=0$. For the other pairs $u, v$ with $u+v=3$, we estimate the first term of (6.5). From $p_{1}, p_{2}, p_{3} \geq 4$ it follows $p_{u} \geq 4$, and thus (6.7) yields $p_{v} \leq(14+k-4 u) / v$ in case $p_{1}+p_{2}+p_{3}=14+k$, and the case $p_{1}+p_{2}+p_{3}=12$ is also covered by putting $k=0$. Moreover, (6.6) yields $y_{v} \geq((2-u) / v) \pi$. Thus

$$
\begin{aligned}
v\left[H\left(p_{v}, y_{v}\right)-c\right] \geq v & {\left[H\left(\frac{14+k-4 u}{v}, \frac{2-u}{v} \pi\right)-c\right] } \\
\geq v & {\left[H\left(\frac{14-4 u}{v}, \frac{2-u}{v} \pi\right)-c\right] } \\
& +k H_{p}\left(\frac{14-4 u}{v}, \frac{2-u}{v} \pi\right) \\
= & \left\{\begin{array}{rrr}
-0.178 & \text { for } u=2, \quad v=1, \\
-0.295-k 0.013 \quad \text { for } u=1, & v=2, \\
-0.279-k 0.039 & \text { for } u=0, & v=3,
\end{array}\right.
\end{aligned}
$$

which together with (6.8) proves Theorem 1 also in this case.

\section{Proof of Theorem 1 in the Remaining Cases, and Proof of Theorem 3(i)}

It remains to prove Theorem 1 in the case of two and three domains. Simultaneously we prove Theorem 3(i), that is, letting $Q(n)$ for each given $n$ be the minimum of the isoperimetric quotients in (1.1), we have to show $Q(2)>3.7614$ and $Q(3)>3.7616$. Using the convexity of the domains, it is easily seen that precisely the topological types of Fig. 4 occur.

Clearly, it is enough to prove (2.5) for $n=2,3$ and with $c$ now being defined as $c:=3.7614$ for $n=2$ and $c:=3.7616$ for $n=3$. Note that $y_{i}<\pi$ for $1 \leq i \leq n$. Since Lemmas 4.1(a) and (b) are a direct consequence of the convexity of $H(p, y)$, they are valid for all positive values of $c$. Thus it remains to show, for $u+v=2$ (resp. 3 ) that

$$
u h_{0}\left[H\left(p_{u}, y_{u}\right)-c\right]+v\left[H\left(p_{v}, y_{v}\right)-c\right]>0
$$
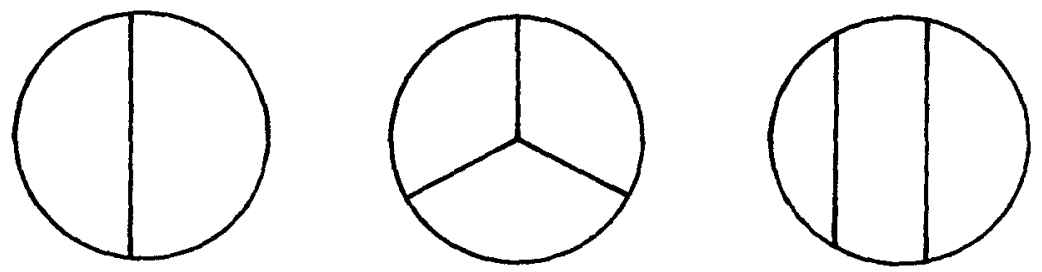

Fig. 4. The topological types of all the decompositions with two and three domains. 
with

$$
u y_{u}+v y_{v}=(u+v-1) \pi .
$$

Note that $p_{u}$ is the mean vertex number of some $u$ domains, and $p_{v}$ is the mean vertex number of the remaining $v$ domains.

One proves (7.1) for each type of Fig. 4 separately; as an example, we describe the procedure for the last type. Consider all pairs of integers $u \geq 0, v \geq 0$ with $u+v=3$. If $u=0$, then $p_{v}=\frac{8}{3}$ is the mean vertex number of all the domains, and (7.2) yields $y_{v}=2 \pi / 3$, so $(7.1)$ is checked immediately. The case $v=0$ is handled in the same way.

In each of the other two cases, $u=1, v=2$ (resp. $u=2, v=1$ ), there are two essentially different possibilities of choosing $u$ domains, and $p_{u}$ is their mean vertex number, while $p_{v}$ is the mean vertex number of the remaining $v$ domains. For each of these four quadruplets $\left(u, v, p_{u}, p_{v}\right)$, the left side of $(7.1)$ is a function of $y_{u}$ because of (7.2), namely,

$$
F\left(y_{u}\right):=u h_{0}\left[H\left(p_{u}, y_{u}\right)-c\right]+v\left[H\left(p_{v}, \frac{2 \pi-u y_{u}}{v}\right)-c\right] .
$$

Note that $y_{v} \leq \pi$, and so (7.2) yields $y_{u} \geq((u-1) / u) \pi$. Dissect the interval $[((u-1) / u) \pi, \pi]$ into intervals small enough. In each such interval, $F\left(y_{u}\right)$ can be estimated from below, since by (3.3) its first summand is increasing in $y_{u}$, and its second is decreasing. In this way, one checks that $F\left(y_{u}\right)>0$ in $[((u-1) / u) \pi, \pi]$, which completes the proof.

\section{Proof of Theorems 2 and 3(ii)}

Formally, the proof of Theorem 2 is completely analogous to that of Theorem 1. Instead of (2.3) we use the estimation

$$
\frac{L_{i}^{2}}{T_{i}} \geq 4\left(\pi-y_{i}+p_{i} \tan \frac{y_{i}}{p_{i}}\right),
$$

which follows from Lhuilier's inequality, see [1]. Consider the function

$$
G(p, y):=4\left(\pi-y+p \tan \frac{y}{p}\right),
$$

again defined on the convex domain $D$ given by $p>0,0 \leq y \leq \pi$, and $y / p<\pi / 2$. In order to prove Theorem 2 , it is enough to show, with $c^{2}=4 \sqrt{12}$, that

$$
T_{1}\left[G\left(p_{1}, y_{1}\right)-c^{2}\right]+\cdots+T_{n}\left[G\left(p_{n}, y_{n}\right)-c^{2}\right]>0,
$$

where $\left(p_{i}, y_{i}\right)$ are points of $D$ satisfying (2.1) and (2.2), and because of (1.4), we may add the condition

$$
g_{0} \leq T_{i} \leq 1 \text { for } 1 \leq i \leq n .
$$

The function $G(p, y)$ has essentialy the same properties as $H(p, y)$ (compare Section 3). Intersecting the function $z=G(p, y)$ with the plane $y=\pi$, we obtain in this 
plane the curve $z=G(p, \pi)=g(p)$ (see Section 1). Since $g(p)=h^{2}(p)$, and $h(p)$ is a convex function for $p>2$, so is $g(p)$. Using this, one shows as in Section 3 that $G(p, y)$ and its partial derivatives have the following properties, for $(p, \pi) \in D$ and $0<t \leq 1$ :

$$
\begin{gathered}
G(t p, t \pi)=4 \pi+t[G(p, \pi)-4 \pi], \\
G_{p}(t p, t \pi)=G_{p}(p, \pi) \text { and } G_{y}(t p, t \pi)=G_{y}(p, \pi), \\
G(p, y) \text { is a convex function, } \\
G_{p}(p, y) \leq 0 \text { and } G_{y}(p, y)>0 \text { for all }(p, y) \in D, \\
G(6, \pi)=g(6)=c^{2}, \\
{\left[G(7, \pi)-c^{2}\right]=-g_{0}\left[G(5, \pi)-c^{2}\right] .}
\end{gathered}
$$

Replacing the function $H(p, y)$ in Lemma 4.1 by $G(p, y)$ and $\sqrt{T_{i}}, c, h_{0}$ by $T_{i}, c^{2}, g_{0}$, respectively, we obtain a lemma whose proof is completely analogous to that of Lemma 4.1. Moreover, Remark 4.2 now allows us to add $g_{0} 4.905=2.707$ to the right side of the inequality corresponding to (4.1).

We use this analogue of Lemma 4.1 to prove Theorem 2 in the case of at least four exterior domains, that is, we show that

$$
\begin{aligned}
\bar{F}\left(u, v ; p_{u}, p_{v}, y_{u}, y_{v}\right):= & u g_{0}\left[G\left(p_{u}, y_{u}\right)-c^{2}\right]+v\left[G\left(p_{v}, y_{v}\right)-c^{2}\right] \\
& +\left(u p_{u}+v p_{v}-5(u+v)+6\right) g_{0}\left[G(5, \pi)-c^{2}\right] \\
> & 0
\end{aligned}
$$

for all integers $u \geq 0$ and $v \geq 0$ with $u+v \geq 4$, and for all points $\left(p_{u}, y_{u}\right)$ and $\left(p_{v}, y_{v}\right)$ of the domain $D$ with

$$
u y_{u}+v y_{v}=(u+v-1) \pi .
$$

In the case where $u, v \neq 0$ we introduce a new variable $t$ by $y_{u}:=t \pi$, and the arising function $\bar{F}\left(u, v ; p_{u}, p_{v}, t\right)$ attains its minimum for $p_{u}=t 5.4620 \ldots, p_{v}=$ $((u+v-1-u t) / v) 6.4699 \cdots$, and $t=1$, as differentiation together with (8.4) and (8.3) shows. In this way,

$$
\bar{F}\left(u, v ; p_{u}, p_{v}, t\right) \geq u 0.341+v 0.347-1.266,
$$

which is, in fact, positive for $u+v \geq 4$.

In the case where $u=0$, it follows from (8.9) that $y_{v}=((v-1) / v) \pi$, and the arising function $\bar{F}\left(v, p_{v}\right)$ attains its minimum for $p_{v}=((v-1) / v) 6.4699 \cdots$. Together with (8.3), we obtain

$$
\bar{F}\left(v, p_{v}\right) \geq v 0.347-1.266,
$$

which is, in fact, positive for $v \geq 4$.

In the case where $v=0$, we obtain in the same way

$$
\bar{F}\left(u, p_{u}\right) \geq u 0.341-0.682,
$$

which is, in fact, positive for $u \geq 4$. 
Next, we prove Theorem 2 in the case of three exterior and at least one interior domains. We proceed as in Section 6, and first tighten the estimations leading to (8.2). It follows from (6.3) that

$$
\frac{L_{4}^{2}}{T_{4}} \geq 4\left(\tan \frac{\pi}{3}+\left(p_{4}-1\right) \tan \frac{2 \pi}{3\left(p_{4}-1\right)}\right) .
$$

The difference between this estimation and that of (8.1) is

$$
\delta\left(p_{4}\right):=4\left(\tan \frac{\pi}{3}+\left(p_{4}-1\right) \tan \frac{2 \pi}{3\left(p_{4}-1\right)}\right)-4 p_{4} \tan \frac{\pi}{p_{4}}
$$

and one obtains

$$
\delta\left(p_{4}=3\right)=0 \quad \text { and } \delta\left(p_{4} \geq 4\right) \geq 0.997 .
$$

Analogously to (6.5), it finally remains to show that, for $u+v=3$,

$$
\begin{aligned}
v\left[G\left(p_{v}, y_{v}\right)-c^{2}\right]+\left(u p_{u}+v p_{v}-9\right) g_{0}\left[G(5, \pi)-c^{2}\right] \\
+ \begin{cases}2.707>0 & \text { if } p_{1}+p_{2}+p_{3}=12, \\
0.550>0 & \text { otherwise, }\end{cases}
\end{aligned}
$$

where

$$
u y_{u}+v y_{v}=2 \pi \text {. }
$$

Recalling that $u p_{u}+v p_{v}=p_{1}+p_{2}+p_{3}=12$ or $14+k$ for $k=0,1,2, \ldots$, we see that the sum of the last two terms of $(8.10)$ is

$$
\begin{aligned}
(12-9) 0.372+2.707 & =3.823 & \text { for } & p_{1}+p_{2}+p_{3}=12 \\
(14+k-9) 0.372+0.550 & =2.410+k 0.372 & \text { for } & p_{1}+p_{2}+p_{3}=14+k
\end{aligned}
$$

Thus, (8.10) is clearly true for $v=0$. For the other pairs $u, v$ with $u+v=3$, we obtain analogously to (6.9), where the case $p_{1}+p_{2}+p_{3}=12$ is covered by $k=0$,

$$
\begin{aligned}
v\left[G\left(p_{v}, y_{v}\right)-c^{2}\right] & \geq v\left[G\left(\frac{14-4 u}{v}, \frac{2-u}{v} \pi\right)-c^{2}\right]+k G_{p}\left(\frac{14-4 u}{v}, \frac{2-u}{v} \pi\right) \\
& = \begin{cases}-1.290 & \text { for } u=2, \quad v=1, \\
-2.150-k 0.090 & \text { for } u=1, \quad v=2, \\
-2.035-k 0.285 & \text { for } u=0, \quad v=3,\end{cases}
\end{aligned}
$$

which together with (8.12) proves Theorem 2 also in this case.

Finally, the same procedure as in Section 7 proves Theorem 3(ii) and so also Theorem 2 in the case of two and three domains.

\section{References}

1. G. Blind, Zerlegung eines konvexen Gebiets in konvexe Gebiete, Arch. Math. 41 (1983), 276-279.

2. G. Fejes Tóth, An isoperimetric quotient for tessellations, Studia Sci. Math. Hungar. 10 (1975), $171-173$. 
3. L. Fejes Tóth, Lagerungen in der Ebene, auf der Kugel und im Raum, Berlin, 1 st Auflage, 1953; 2nd Auflage, 1972.

4. L. Fejes Tóth, Isoperimetric problems for tilings, Mathematika 32 (1985), 10-15.

5. G. Kertész, A counterexample to an isoperimetric problem of L. Fejes Tóth, Studia Sci. Math. Hungar. 24 (1989), 303-304.

6. E. Vásárhelyi, An isoperimetric problem for tilings, Intuitive Geometry (Siófok, 1985), pp. 573-602. Colloq. Math. Soc. János Bolyai, 48, North-Holland, Amsterdam, 1987.

7. E. Vásárhelyi, Ein isoperimetrisches Problem bezüglich Zerlegungen der euklidischen Ebene, Studia Sci. Math. Hungar. 27 (1992), 287-294.

Received April 21, 1999, and in revised form June 21, 2000. Online publication January 17, 2001. 